\title{
Predictive value of angiogenesis-related gene profiling in patients with HER2-negative metastatic breast cancer treated with bevacizumab and weekly paclitaxel
}

\author{
Marta Mendiola ${ }^{1}$, Virginia Martínez-Marin ${ }^{2,3}$, Jesús Herranz ${ }^{4}$, Victoria Heredia ${ }^{1}$, \\ Laura Yébenes ${ }^{5}$, Pilar Zamora ${ }^{2,3}$, Beatriz Castelo ${ }^{2,3}$, Álvaro Pinto ${ }^{2,3}$, María Miguel ${ }^{1}$, \\ Esther Díaz ${ }^{3}$, Angelo Gámez³, Juan Ángel Fresno ${ }^{3}$, Ana Ramírez de Molina4, David \\ Hardisson $^{1,5}$, Enrique Espinosa ${ }^{2,3}$, Andrés Redondo ${ }^{2,3}$ \\ ${ }^{1}$ Molecular Pathology and Therapeutic Targets Group, La Paz University Hospital - IdiPAZ, Madrid, Spain \\ ${ }^{2}$ Department of Medical Oncology, La Paz University Hospital, Madrid, Spain \\ ${ }^{3}$ Translational Oncology Group, La Paz University Hospital - IdiPAZ, Madrid, Spain \\ ${ }^{4}$ IMDEA, Campus de Excelencia Internacional CEI (UAM-CSIC), Madrid, Spain \\ ${ }^{5}$ Department of Pathology, La Paz University Hospital, Madrid, Spain \\ Correspondence to: Andrés Redondo, e-mail: aredondos@uam.es \\ Keywords: metastatic breast carcinoma, bevacizumab and weekly paclitaxel, predictive, angiogenesis, gene expression \\ Received: November 29, 2015 \\ Accepted: February 25, 2016 \\ Published: March 16, 2016
}

\section{ABSTRACT}

Bevacizumab plus weekly paclitaxel improves progression-free survival (PFS) in HER2-negative metastatic breast cancer $(\mathrm{mBC})$, but its use has been questioned due to the absence of a predictive biomarker, lack of benefit in overall survival (OS) and increased toxicity. We examined the baseline tumor angiogenic-related gene expression of 60 patients with $\mathrm{mBC}$ with the aim of finding a signature that predicts benefit from this drug.

Multivariate analysis by Lasso-penalized Cox regression generated two predictive models: one, named G-model, including 11 genes, and the other one, named GCmodel, including 13 genes plus 5 clinical covariates. Both models identified patients with improved PFS (HR (Hazard Ratio) 2.57 and 4.04, respectively) and OS (HR 3.29 and 3.43, respectively). The G-model distinguished low and high risk patients in the first 6 months, whereas the GC-model maintained significance over time.

\section{INTRODUCTION}

Breast cancer is a heterogeneous disease regarding molecular and clinical features. In the metastatic setting, the expression of human epidermal growth factor receptor 2 (HER2) and hormonal receptors determine the selection of therapies. Treatment for HER-2 negative mBC includes hormonal therapy, chemotherapy and bevacizumab combined regimens.

In the pivotal E2100 study, bevacizumab plus weekly paclitaxel increased overall response rate $(50 \%$ vs $22 \%$ ) and progression-free survival (PFS) (median of 11.8 vs 5.9 months) compared to paclitaxel alone [1]. Other phase III trials in $\mathrm{mBC}$ also reported a PFS benefit with the addition of bevacizumab to chemotherapy, both in first $[2,3]$ and second line therapies [4]. However, none of them showed a significant improvement in overall survival (OS), possibly due to the confounding effect of post-progression therapy, lack of statistical power, or treatment crossover. This, along with economic issues, lack of validated biomarkers and increased toxicity has put into question the role of bevacizumab in $\mathrm{mBC}$. Nowadays, the identification of predictive biomarkers for this drug remains a challenge.

Technological improvements in molecular profiling have allowed a better knowledge of breast cancer biology, leading to the development of new tests that help in clinical decision making.

In the present study we analysed a set of 168 genes related to angiogenesis and other processes on a discrete series of patients treated with bevacizumab and weekly paclitaxel. Our aim was to find a molecular signature predicting PFS benefit from this regimen. To date, this is the first report of a biomarker profile with PFS prediction. 
Table 1: Patients characteristics

\begin{tabular}{|c|c|}
\hline Patients characteristics & $\mathbf{N}(\%)$ \\
\hline \multicolumn{2}{|l|}{ Estrogen Receptor (ER) } \\
\hline Positive & $49(81.7)$ \\
\hline Negative & $11(18.3)$ \\
\hline \multicolumn{2}{|l|}{ Progesterone receptor (PR) } \\
\hline Positive & $41(68.3)$ \\
\hline Negative & $19(31.7)$ \\
\hline \multicolumn{2}{|l|}{ IHQ subtype } \\
\hline Triple negative & $11(18.3)$ \\
\hline ER positive, $P R$ negative & $8(13.3)$ \\
\hline ER positive, $\mathrm{PR}$ positive & $41(68.3)$ \\
\hline \multicolumn{2}{|l|}{ Adjuvant chemotherapy } \\
\hline Yes & $46(76.7)$ \\
\hline No & $14(23.3)$ \\
\hline \multicolumn{2}{|l|}{ Disease-free interval } \\
\hline$\leq 24$ months & $17(28.3)$ \\
\hline$>24$ months or stage IV at diagnosis & $43(71.7)$ \\
\hline \multicolumn{2}{|l|}{ Number of chemo lines for metastasic disease } \\
\hline 0 & $36(60)$ \\
\hline$>1$ & $24(40)$ \\
\hline \multicolumn{2}{|l|}{ Previous chemotherapy } \\
\hline None (or non antracyclines: e.g. CMF) & $15(25)$ \\
\hline Anthracyclines & $17(28.3)$ \\
\hline Anthracyclines and taxanes & $28(46.7)$ \\
\hline \multicolumn{2}{|l|}{ Metastatic locations } \\
\hline 1 or 2 & $27(45)$ \\
\hline$\geq 3$ or hepatic involvement & $33(55)$ \\
\hline
\end{tabular}

IHQ: inmunohistochemistry CMF: old chemotherapy regimen

We selected genes implicated in angiogenesis and other related processes, such as Epithelial to Mesenchymal Transition (EMT) and inflammation, that could have an impact in the response or resistance to bevacizumab [5].

\section{RESULTS}

\section{Descriptive statistics and univariate analysis}

Sixty patients were included and their clinical characteristics are summarized in Table 1. Median age at diagnosis was 54 years (range 33-76). Almost half of the patients had received prior therapy with anthracyclines and taxanes, and $40 \%$ had been treated with one or more previous lines of chemotherapy for metastatic disease.

Forty-five percent of the patients achieved a partial response and $13 \%$ a complete response. Median PFS was 11.4 months (range 0.5-46.1), and median OS was 29 months (range 1.41-44.8). All patients received bevacizumab until progression, but paclitaxel had to be discontinued after 6-8 cycles in 32 patients, mostly due to toxicity. During the bevacizumab continuation phase, 15 patients (53.5\% of estrogen receptor -ER- positive patients with continuation treatment) also received hormonal therapy. 
Table 2: Univariate analysis of clinical variables for progression-free survival

\begin{tabular}{|c|c|c|}
\hline Variable & HR $(95 \%$ CI) & $P$ value \\
\hline Age & $1.02(0.99-1.04)$ & 0.18 \\
\hline $\begin{array}{l}\text { Disease-free interval } \\
(\geq 24 \text { vs }<24 \text { months })\end{array}$ & $0.49(0.27-0.89)$ & 0.02 \\
\hline $\begin{array}{l}\text { Estrogen-receptor } \\
\text { (positive vs negative) }\end{array}$ & $0.42(0.21-0.83)$ & 0.02 \\
\hline $\begin{array}{l}\text { Progesterone-receptor } \\
\text { (positive vs negative) }\end{array}$ & $0.58(0.32-1.03)$ & 0.07 \\
\hline $\begin{array}{l}\text { Adjuvant chemotherapy } \\
\text { (yes vs no) }\end{array}$ & $0.69(0.37-1.27)$ & 0.25 \\
\hline Prior anthracyclines and taxanes (yes vs no) & $1.17(0.63-2.17)$ & 0.62 \\
\hline Prior lines for metastatic disease ( $\geq 1$ vs 0 ) & $1.64(0.93-2.87)$ & 0.09 \\
\hline $\begin{array}{l}\text { Metastatic location } \\
(<3 \text { locations and no liver involvement vs } \\
\geq 3 \text { or hepatic })\end{array}$ & $2.92(1.61-5.32)$ & 0.0003 \\
\hline Hormonal therapy* & $0.48(0.2-1.17)$ & 0.11 \\
\hline
\end{tabular}

*Only in subgroup of patients with bevacizumab continuation treatment and ER positive

Univariate analysis of clinical and pathological variables for PFS is presented in Table 2. Disease-free interval (DFI), ER and metastatic location (3 or more locations or hepatic involvement) were significantly associated with PFS. Summary information for the gene expression measurements as well as the Hazard Ratio (HR) for the association between the gene expression values and PFS is presented on Supplementary Table. Nine genes (CDH11, ESR1, FABP5, FN1, IL8, NOTCH3, PGR, $P L A U$ and $S L C 39 A 6$ ) showed adjusted $\mathrm{p}$ values $<0.05$ for association with cancer progression.

\section{Multivariate cox models}

A multivariate analysis with five clinical variables (DFI, ER, ML, prior anthracyclines and taxanes, and prior chemotherapy treatment for metastatic disease) was associated with PFS (Table 3). This was defined as the clinical or C-model.

We then combined clinical and gene variables into two models using the least absolute shrinkage and selection operator (LASSO) [6] [7] for variable selection: one model had gene expression only (G-model), and the other had both gene expression and clinical variables (GCmodel). The G-model included 11 genes (SLC39A6, REL, IL8, FN1, PLAU, HES1, HMBS, DDIT4, FABP5, ACVRL1, $P G R$ ) (Table 4). The GC-model consists of 13 genes (REL, FN1, NOTCH3, DDIT4, IL8, ADRBK1, FABP5, PLAU, $H M B S, P T K 2 B, T H B S 1, S L C 39 A 6, T C F 3)$ and the five clinical variables mentioned previously (Table 5). The beta coefficient signs of the genes agreed with the expression results from the univariate analysis. Figure 1 shows Kaplan Meier plots for progression-free survival (PFS) and overall survival (OS) in G and GC models. The HR, differences in median and $\mathrm{p}$ values for these predictions are summarized in Tables 6 (PFS) and 7(OS).

\section{Comparison of accuracy of prediction of the models through roc curves}

Models were also compared to evaluate the gain of accuracy by time-dependent ROC curves [8], and results are shown in Figure 2. In the first 6 months $\mathrm{G}$ and GC models worked better than $\mathrm{C}$ model (AUC 0.68 and 0.72 versus AUC 0.53 respectively). The $\mathrm{G}$ model lose accuracy with longer follow-up, whereas GC remained accurate over time, as shown in Figures $2 \mathrm{c}$ and $2 \mathrm{~d}$.

\section{Inmunohistochemistry evaluation of biomarkers}

Tissue microarrays (TMA) were used to evaluate the protein expression encoded by those genes with the higher beta coefficients: cREL, SLC39A6, NOTCH, FN1 and DDIT4. A summary of these studies is shown in Figure 3. None of the proteins analyzed were independently associated with PFS.

\section{DISCUSSION}

Bevacizumab was approved as first-line treatment for $\mathrm{mBC}$ in 2008. Three years later, the Food and Drug Administration revoked the indication due to increased 
Table 3: Clinical multivariate analysis for progression-free survival (C-model)

\begin{tabular}{lcc}
\hline Variable & HR (CI 95\%) & P value \\
\hline $\begin{array}{l}\text { Disease-free interval } \\
(\geq 24 \text { vs }<24 \text { months) }\end{array}$ & $0.74(0.35-1.59)$ & 0.43 \\
$\begin{array}{l}\text { Estrogen-receptor } \\
\text { (positive vs negative) }\end{array}$ & $0.41(0.17-1.03)$ & 0.06 \\
$\begin{array}{l}\text { Prior anthracyclines and taxanes } \\
\text { (yes vs no) }\end{array}$ & $1.02(0.52-2.01)$ & 0.94 \\
$\begin{array}{l}\text { Prior lines for metastatic disease } \\
(\geq 1 \text { vs } 0)\end{array}$ & $1.55(0.8-3)$ & 0.19 \\
$\begin{array}{l}\text { Metastatic location } \\
(<3 \text { locations and no liver involvement }\end{array}$ & & 0.0005 \\
vs $\geq 3$ or hepatic) & $3.07(1.63-5.8)$ & \\
\hline
\end{tabular}

Table 4: Genetic model (G-model)

\begin{tabular}{lcc}
\hline Gene & Beta coefficient (LASSO) & HR (IC95\%) \\
\hline SLC39A6 & 0,289 & $1,72(1,28-2,3)$ \\
REL & $-0,282$ & $0,74(0,56-0,99)$ \\
IL8 & $-0,231$ & $0,7(0,54-0,89)$ \\
FN1 & 0,191 & $1,51(1,18-1,93)$ \\
PLAU & 0,152 & $1,5(1,14-1,98)$ \\
HES1 & 0,138 & $1,15(0,92-1,44)$ \\
HMBS & $-0,063$ & $0,81(0,64-1,04)$ \\
DDIT4 & 0,049 & $1,14(0,87-1,51)$ \\
FABP5 & $-0,043$ & $0,72(0,55-0,96)$ \\
ACVRL1 & 0,031 & $1,32(0,99-1,77)$ \\
PGR & 0,021 & $1,46(1,09-1,95)$ \\
\hline
\end{tabular}

toxicity without a clear benefit in OS. However, the European Medicines Agency decided to keep the indication, but only in combination with paclitaxel (regimen with the most benefit in SLP).

The identification of a reliable biomarker would avoid unnecessary toxicity in non-responders, reduce healthcare costs, and therefore improve cost-effectiveness. VEGF is a master regulator of angiogenesis, and should be expected to predict response to bevacizumab, but the results of different studies are controversial. Translational studies associated to bevacizumab trials in $\mathrm{mBC}$ have shown a possible role of plasmatic VEGFA (pVEGFA) and VEGFR2 levels as response predictors [9-12]. However, preliminary results of the prospective study Meridian showed that baseline pVEGFA levels were not associated to improved PFS [13].
Due to the high number of genes involved in angiogenesis, a single biomarker is unlikely to predict benefit from bevacizumab. Therefore, identification of alternative biomarkers is a main subject for translational oncology research, and, to our knowlegde, this is the first report of a biomarker profile predicting PFS benefit in $\mathrm{mBC}$ patients treated with bevacizumab and weekly paclitaxel.

We selected a group of genes described to have a role in angiogenesis and other related processes, to investigate if any combination could impact on response to bevacizumab [5]. In the multivariate analysis we decided to include clinical factors found to be relevant in another study with bevacizumab-containing therapy [14]. Some of these factors did not reach statistical significance, probably due to the small sample size. 
Table 5: Combined genetic and clinical model (GC-model)

\begin{tabular}{lcc}
\hline Variable & Beta (LASSO) & HR \\
\hline Disease-free interval & -0.223 & 0.8 \\
( $\geq 24$ vs $<24$ months) & & 0.4 \\
Estrogen-receptor & & \\
(positive vs negative) & -0.907 & 1.014 \\
Prior anthracyclines and taxanes (yes vs & & \\
no) & 0.008 & 1.57 \\
Prior lines for metastatic disease ( $\geq 1$ vs & & \\
0) & 0.45 & 4.52 \\
Metastatic location & & \\
(<3 locations and no liver involvement & 1.509 & 0.71 \\
vs $\geq 3$ or hepatic) & & 1.38 \\
REL & -0.349 & 1.33 \\
FN1 & 0.322 & 1.32 \\
NOTCH3 & 0.287 & 0.8 \\
DDIT4 & 0.281 & 0.81 \\
IL8 & -0.223 & 0.84 \\
ADRBK1 & -0.21 & 1.17 \\
FABP5 & -0.176 & 0.86 \\
PLAU & 0.159 & 1.15 \\
HMBS & -0.155 & 1.12 \\
PTK2B & 0.136 & 0.97 \\
THBS1 & 0.114 & \\
SLC39A6 & 0.05 & \\
TCF3 & -0.028 & \\
\hline
\end{tabular}

This is the main weakness of our study, along with the absence of a validation set. Validation was not feasible because of the limited number of patients treated with bevacizumab and paclitaxel. Possible biases are difficult to be controlled in a retrospective study, including the use of chemotherapy before bevacizumab and the use of hormonal therapy during the bevacizumab continuation phase in some patients. The latter group was not included in the multivariate analysis.

On the other hand, the strengths of our work are the biological plausibility of the selected genes, the low number of genes included in the models, and the statistical method that offers a robust internal validation.

The G-model consists of 11 genes, and the GCmodel includes 5 clinical covariates and 13 genes. Among the genes, 8 are present in both models: SLC39A6, REL, IL8; FN1, PLAU, HES1, HMBS, DDIT4, FABP5, $A C V R L 1$, and $P G R$. The sign of beta coefficients agrees in all cases with the expression results of the univariate analysis of the genes, and is maintained in both models.
SLC39A6 and REL in the G-model, and FN1 and, again, $R E L$ in the GC-model had the highest weight.

SLC39A6 expression has been described in clinical breast-tumour populations as significantly associated with the estrogen receptor status [15]. It has also been associated with the spread of breast cancer to regional lymph nodes $[16,17]$. Interestingly, this gene had the highest beta coefficient in the G-model, but one of the lowest in the GC one, where ER is also included.

FN1 has been previously described as part of an extracellular matrix gene cluster associated with resistance to first-line tamoxifen therapy in patients with $\mathrm{mBC}$ and with the development of metastasis [18] [19].

$R E L$ is part of the nuclear factor-карраB $(N F-\kappa B)$ complexes. It had the highest beta coefficient in both models, being associated with improved PFS and OS. The involvement of $N F-\kappa B$ in neoplastic proliferation of human breast cancer cells has been described under estrogen-free conditions in vitro, where it induces additive anticancer effects with tamoxifen [20]. Its specific role 
in antiangiogenic therapy response should be further characterized.

We used LASSO Cox regression model to determine genes significantly related to PFS. This model accounts for overfitting, a problem inherent to studies where the number of genes far exceeds the number of patients. The output is a profile with a reduced number of genes, which makes it more suitable for clinical application. We also used LOOCV method, and a permutation test to assess the statistical significance of the models. Both GC and G models fared better than $\mathrm{C}$ model, being correlated with PFS and OS (Table 7).
We also tested the predictive accuracy of these models by time-dependent ROC curves (Figure 2). The relative weight of the gene component in $\mathrm{G}$ and GC models remained constant over time, whereas the clinical variables were important in the long term. A plausible explanation is that clinical parameters are purely prognostic, whereas genes predict benefit from treatment, so their influence is detected earlier.

In summary, we described two models that predict improved PFS and OS with bevacizumab-paclitaxel therapy: the G-model included a combination of 11 genes, and the (GC-model consisted of 13 genes and 5 clinical

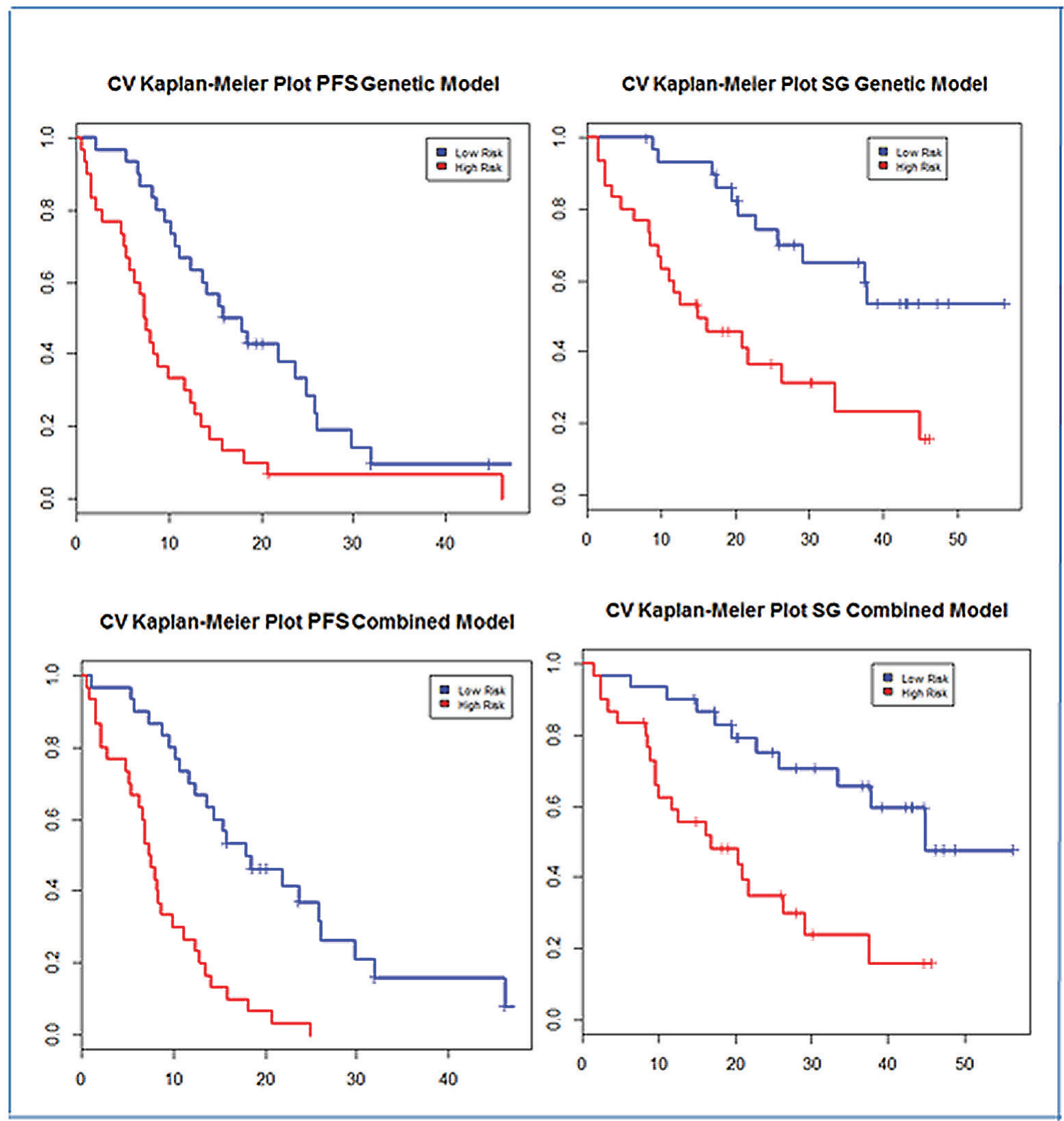

Figure 1: Progression-free survival and overall survival Kaplan-Meier curves of the two groups established by the G-model (a and b) and by the GC-model (c and d). 
Table 6: Hazard ratio, medians of progression-free survival and p-values of the two groups of patients established by the $G$ and $G C$ models

\begin{tabular}{lcccc}
\hline Model & Risk Group & HR (CI 95\%) & p-value & $\begin{array}{c}\text { Median PFS } \\
(\mathbf{m o n t h s )}\end{array}$ \\
\hline \multirow{2}{*}{ G-model } & Low-risk & 1 & 0,048 & $16,9(12,3-25,8)$ \\
& High-risk & $2.57(1.47-4.48)$ & $7,4(5,7-12,4)$ \\
GC-model & Low-risk & 1 & $<0,001$ & $17,9(13,7-29,8)$ \\
& High-risk & $4.04(2.2-7.44)$ & $7,4(6,2-11,1)$ \\
\hline
\end{tabular}

Table 7: Hazard ratio, medians of overall survival and p-values of the two groups of patients established by the $G$ and GC models

\begin{tabular}{lcccc}
\hline Model & Risk Group & HR (CI 95\%) & p-value & $\begin{array}{c}\text { Median PFS } \\
\text { (months) }\end{array}$ \\
\hline G-model & Low-risk & 1 & 0,001 & NR \\
& High-risk & $3.29(1.57-6.91)$ & & $14,9(9,9-44,8)$ \\
\multirow{2}{*}{ GC-model } & Low-risk & 1 & $<0,001$ & $44,8(33,3-\mathrm{NR})$ \\
& High-risk & $3.43(1.62-7.26)$ & & $16,8(9,9-37,4)$ \\
\hline
\end{tabular}

variables. Both had good accuracy in the first six months, whereas the GC-model remained accurate over time.

These findings should be evaluated in larger independent series in order to develop a routine clinical test to predict the benefit of bevacizumab in $\mathrm{mBC}$ patients.

\section{MATERIALS AND METHODS}

\section{Patient selection and tumor sample collection}

The study was approved by our institutional Ethics Committee. Patients with a diagnosis of HER2negative mBC between 2007 and 2011 and treated with bevacizumab and paclitaxel were identified from local records. The schedule consisted of bevacizumab $10 \mathrm{mg} /$ $\mathrm{m} 2$ days 1 and 15 plus weekly paclitaxel $80 \mathrm{mg} / 2$ days 1 , 8 and 15 , on a 28 -day cycle. REMARK criteria were used to help in patient selection [21].

Seventy eight patients fulfilled the inclusion criteria. Six of them were excluded due to the absence of primary tumor biopsy, and another 12 because of poor quality material. The study included the primary tumor from 60 patients. All of them had a minimum follow-up of 18 months since the beginning of the treatment (unless progression and/or death due to $\mathrm{mBC}$ occurred before). Patients with an early withdrawal due to toxicity were not included.

The variable selected to generate a gene signature was progression-free survival, defined as the time from the first administration of bevacizumab-paclitaxel treatment to the first evidence of relapse, death or last record available dates. Other outcome variables, such as response to treatment and overall survival, were also evaluated but were not used to develop the molecular signature.

\section{Gene expression analysis}

\section{RNA purification from FFPE samples}

Sixty archival FFPE cases were evaluated by two breast cancer expert pathologists. Regions of invasive carcinoma were confirmed, and different areas with more than $80 \%$ of malignant epithelial cells were selected. Four to eight $\mu \mathrm{m}$ sections were used for total RNA isolation, with MasterPure RNA Purification Kit (EPICENTRE Biotechnologies, Madison, WI, USA) according to the manufacturer's instructions with minor modifications. RNA concentrations and quality were measured using a Nanodrop 1000A spectrophotometer (Nanodrop Technologies, Wilmington, DE, USA).

\section{Real-Time Quantification of Gene Expression}

One $\mu \mathrm{g}$ of total RNA was used for cDNA synthesis with the High Capacity Archive cDNA Reverse Transcription kit (Applied Biosystems, Foster City, CA, USA), and performed in an Applied Biosystems 7900 thermal cycler for $10 \mathrm{~min}$ at $25^{\circ} \mathrm{C}, 120 \mathrm{~min}$ at $37^{\circ} \mathrm{C}$, and then held at $4^{\circ} \mathrm{C}$.

The RT obtained products were amplified using a Real Time Ready Custom panel 384 (Hoffmann-La 
Roche, Basel, Switzerland). This platform enables the quantitation of 168 genes (Supplementary Table) plus 19 housekeeping (HK) genes and 5 internal controls per sample. The reactions were performed on the LightCycler ${ }^{\circledR}$ 480 as follows: an initial step at $95^{\circ} \mathrm{C}$ for $10 \mathrm{~min}, 45$ amplification cycles with $10 \mathrm{~s}$ at $95^{\circ} \mathrm{C}, 30 \mathrm{~s}$ at $60^{\circ} \mathrm{C}$, and $1 \mathrm{~s}$ at $72^{\circ} \mathrm{C}$, and a final cooling step at $40^{\circ} \mathrm{C}$ for $30 \mathrm{~s}$.

\section{Data processing}

168 candidate genes were selected from the literature as related with the angiogenic process and other progression and resistance mechanisms, such as EMT or inflammation.

Nineteen housekeeping (HK) genes were also included in the study (Supplementary Table 1). Twelve of

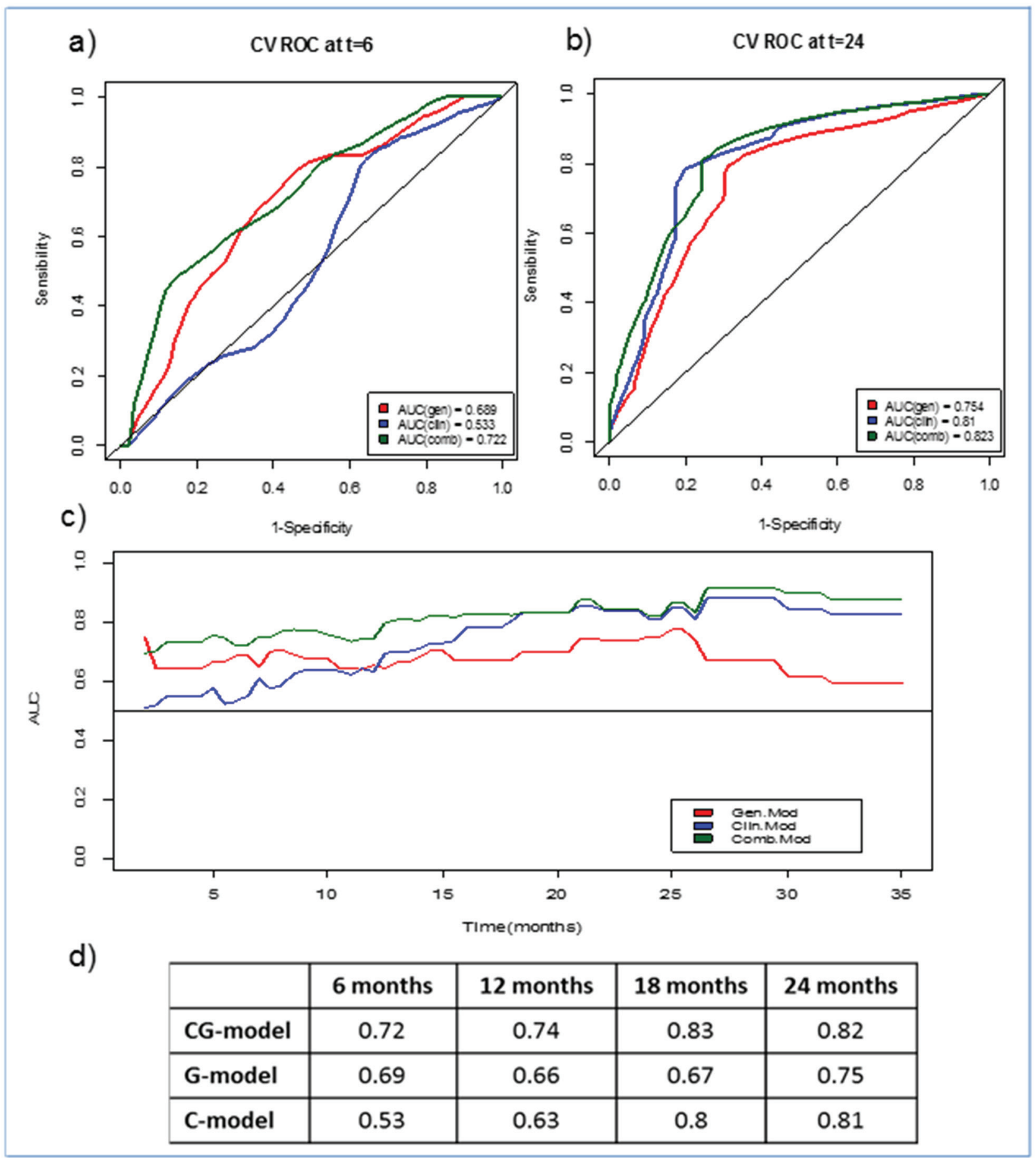

Figure 2: Time-dependent ROC curves for the three models (GC, G and C). a. At 6 months, b. At 24 months, c. AUC evolution for each model over time, $\mathbf{d}$. AUC values for each model at four specific time points 
them were selected by GeNorm software [22] and used for the normalization factor applied to the raw data to get the relative expression value by the DDCt.

\section{Statistical analysis}

Univariate Cox regression models were fitted to evaluate the association between continuous RNA expression values and clinical covariates with survival.

The prediction models were built with penalized multivariate Cox regression proportional hazards modelling using L1-penalized (Lasso). A cross-validated (CV) risk score was calculated by leave-one-out crossvalidation (LOOCV) for each model $[6,7]$. Patients were classified into high- and low-risk groups, based on the median value of the cross-validated risk score, and crossvalidated Kaplan-Meier curves (CV KM) were estimated. A log-rank statistic was computed for the CV KM plots and the statistical significance was evaluated based on the permutation distribution of the cross-validated logrank-statistic, repeating the whole LOOCV process with randomly permuted survival times and censoring indicators. Cross-validated time dependent receiver operating characteristics (ROC) curves were computed using the cross-validated risk scores. The area under the curve (AUC) values calculated from the CV ROC curves were used as measure of predictive accuracy of the models [8]. All statistical analyses were performed using $\mathrm{R}$ software (version 3.1.1) and the analysis was conducted by the penalized and survival ROC packages in R software.

\section{Inmunohistochemistry analysis}

Genes found to be related to PFS in the multivariate analysis were further analyzed by tissue microarray. Representative areas of the tumors were selected on hematoxylin and eosin-stained sections and marked on individual paraffin blocks by an expert pathologist in the field. Two tissue cores ( $1 \mathrm{~mm}$ in diameter) were obtained from each specimen. The tissue cores were arrayed into a receptor paraffin block using a TMA workstation (Beecher Instruments, Silver Spring, MD, USA), as described previously [23]. A hematoxylin and eosin-stained section of the array was reviewed to confirm the presence of

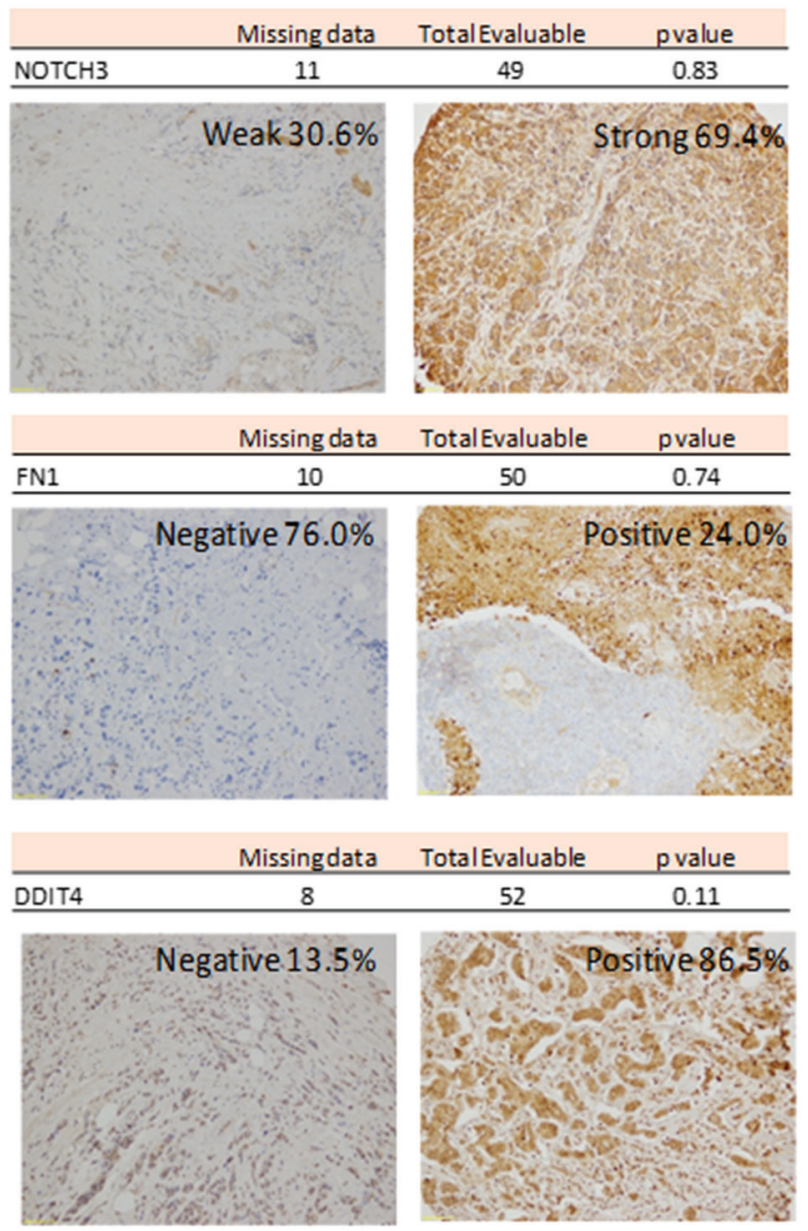

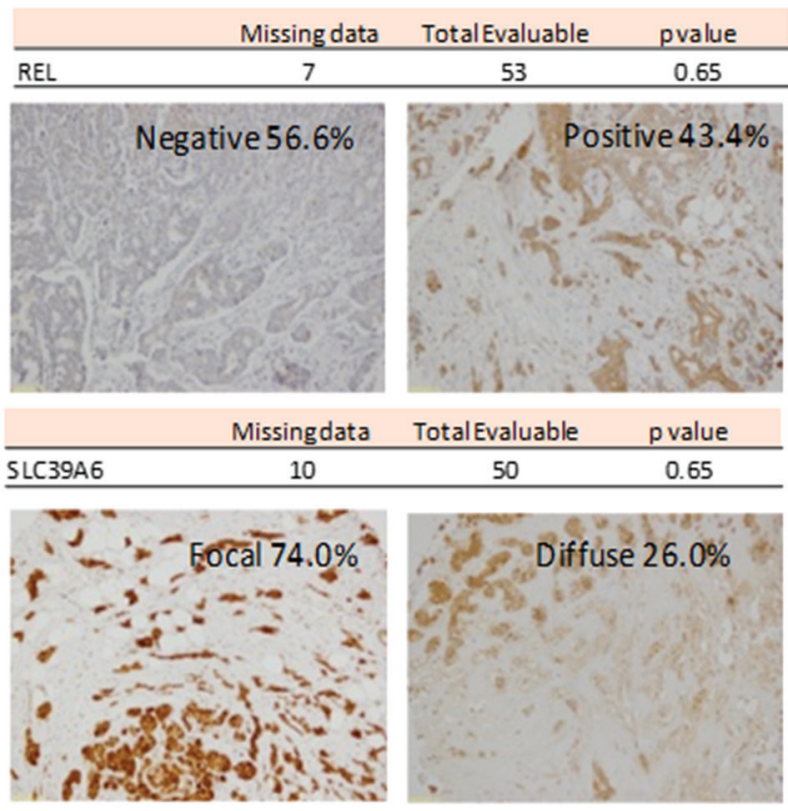


morphologically representative areas of the original lesions.

Immunohistochemistry (IHC) was performed as described previously [24] on 4um sections of the TMAs, obtained by a semiautomated microtome HM3508 (Microm). Briefly, the tissue sections were deparaffinized and rehydrated in water, after which antigen retrieval was carried out in a DAKO PT Link in citrate buffer $(\mathrm{pH}=6)$. Endogenous peroxidase and nonspecific antibody reactivity was blocked with peroxidase blocking reagent (Dako, Glostrup, Denmark) at room temperature for $15 \mathrm{~min}$.

The sections were then incubated for $60 \mathrm{~min}$ with the following antibodies: rabbit monoclonal cMET (SP44, \#790-4430, Hoffmann-La Roche Ltd, Basel, Switzerland) and cREL (EPR2258, \#ab108299, Abcam, Cambridge, UK); rabbit polyclonal: FN1 (\#A0245, Dako, Glostrup, Denmark), SLC39A6 (\#ab61307), DDIT4 (\#ab63059), and NOTCH3 (\#ab60087), all these last from Abcam (Cambridge, UK). Detection was performed with Envision Plus Detection System (Dako, Glostrup, Denmark).

Cytoplasmic staining for cREL and SLC39A6, as well as nuclear and cytoplasmic staining for DDIT4 was classified as negative, focal or diffuse. NOTCH3 presence was diffuse when present, so we evaluated absence or weak/strong staining. Stromal FN1 staining observed in the stromal component was also evaluated. The pathologists performing the immunohistochemistry analysis were blinded for patient's outcome.

\section{ACKNOWLEDGMENTS AND FUNDING}

This work was supported by a grant from the Independent Clinical Research Program (EC10-342), ISCIII (Instituto de Salud Carlos III), Spanish Ministry of Health, and funded also from Roche Farma S.A.U.

\section{CONFLICTS OF INTEREST}

The authors declare no conflicts of interest, with the exception of Dr. Redondo (honoraria, advisory role and research funding from Roche Farma).

\section{REFERENCES}

1. Miller K, Wang M, Gralow J, Dickler M, Cobleigh M, Perez EA, Shenkier T, Cella D, Davidson NE. Paclitaxel plus bevacizumab versus paclitaxel alone for metastatic breast cancer. The New England journal of medicine. 2007; 357:2666-2676.

2. Miles DW, Chan A, Dirix LY, Cortes J, Pivot X, Tomczak P, Delozier T, Sohn JH, Provencher L, Puglisi F, Harbeck N, Steger GG, Schneeweiss A, Wardley AM, Chlistalla A, Romieu G. Phase III study of bevacizumab plus docetaxel compared with placebo plus docetaxel for the first-line treatment of human epidermal growth factor receptor 2-negative metastatic breast cancer. Journal of clinical oncology. 2010; 28:3239-3247.

3. Robert NJ, Dieras V, Glaspy J, Brufsky AM, Bondarenko I, Lipatov ON, Perez EA, Yardley DA, Chan SY, Zhou X, Phan SC, O'Shaughnessy J. RIBBON-1: randomized, double-blind, placebo-controlled, phase III trial of chemotherapy with or without bevacizumab for first-line treatment of human epidermal growth factor receptor 2-negative, locally recurrent or metastatic breast cancer. Journal of clinical oncology. 2011; 29:1252-1260.

4. Brufsky AM, Hurvitz S, Perez E, Swamy R, Valero V, O'Neill V, Rugo HS. RIBBON-2: a randomized, doubleblind, placebo-controlled, phase III trial evaluating the efficacy and safety of bevacizumab in combination with chemotherapy for second-line treatment of human epidermal growth factor receptor 2-negative metastatic breast cancer. Journal of clinical oncology. 2011; 29:4286-4293.

5. Carbone C, Moccia T, Zhu C, Paradiso G, Budillon A, Chiao PJ, Abbruzzese JL, Melisi D. Anti-VEGF treatmentresistant pancreatic cancers secrete proinflammatory factors that contribute to malignant progression by inducing an EMT cell phenotype. Clinical cancer research. 2011; 17:5822-5832.

6. Simon RM, Subramanian J, Li MC, Menezes S. Using cross-validation to evaluate predictive accuracy of survival risk classifiers based on high-dimensional data. Briefings in bioinformatics. 2011; 12:203-214.

7. Bovelstad HM, Nygard S, Borgan O. Survival prediction from clinico-genomic models--a comparative study. BMC bioinformatics. 2009; 10:413.

8. Heagerty PJ, Lumley T, Pepe MS. Time-dependent ROC curves for censored survival data and a diagnostic marker. Biometrics. 2000; 56:337-344.

9. Miles DW, de Haas SL, Dirix LY, Romieu G, Chan A, Pivot X, Tomczak P, Provencher L, Cortes J, Delmar PR, Scherer SJ. Biomarker results from the AVADO phase 3 trial of first-line bevacizumab plus docetaxel for HER2negative metastatic breast cancer. British journal of cancer. 2013; 108:1052-1060.

10. Gianni L, Romieu GH, Lichinitser M, Serrano SV, Mansutti M, Pivot X, Mariani P, Andre F, Chan A, Lipatov O, Chan S, Wardley A, Greil R, Moore N, Prot S, Pallaud C, et al. AVEREL: a randomized phase III Trial evaluating bevacizumab in combination with docetaxel and trastuzumab as first-line therapy for HER2-positive locally recurrent/metastatic breast cancer. Journal of clinical oncology. 2013; 31:1719-1725.

11. Cameron D, Brown J, Dent R, Jackisch C, Mackey J, Pivot X, Steger GG, Suter TM, Toi M, Parmar M, Laeufle R, Im YH, Romieu G, Harvey V, Lipatov O, Pienkowski T, et al. Adjuvant bevacizumab-containing therapy in triplenegative breast cancer (BEATRICE): primary results of a randomised, phase 3 trial. The Lancet Oncology. 2013; 14:933-942. 
12. Dos Santos LV, Cruz MR, Lima Lopes G, Lima JP. VEGF-A levels in bevacizumab-treated breast cancer patients: a systematic review and meta-analysis. Breast cancer research and treatment. 2015; 151:481-489.

13. Miles D CD, Bondarenko I, Lyudmila M, Alcedo JC, Lopez RI, Im SA, Canon JL, Shparyk Y, Yardley D, Masuda N, Ro J, Hubeaux S, et al. First results from the double-blind placebo (PL)-controlled randomised phase III MERiDiAN trial prospectively evaluating plasma (p)VEGF-A in patients (pts) receiving first-line paclitaxel (PAC) +/- bevacizumab (BV) for HER2-negative metastatic breast cancer (mBC). Abstract 1866 European Cancer Congress- Viena 2015.

14. Llombart-Cussac A, Pivot X, Biganzoli L, Cortes-Funes H, Pritchard KI, Pierga JY, Smith I, Thomssen C, Srock S, Sampayo M, Cortes J. A prognostic factor index for overall survival in patients receiving first-line chemotherapy for HER2-negative advanced breast cancer: an analysis of the ATHENA trial. Breast. 2014; 23:656-662.

15. McClelland RA, Manning DL, Gee JM, Willsher P, Robertson JF, Ellis IO, Blamey RW, Nicholson RI. Oestrogen-regulated genes in breast cancer: association of pLIV1 with response to endocrine therapy. British journal of cancer. 1998; 77:1653-1656.

16. Manning DL, Robertson JF, Ellis IO, Elston CW, McClelland RA, Gee JM, Jones RJ, Green CD, Cannon $\mathrm{P}$, Blamey RW, et al. Oestrogen-regulated genes in breast cancer: association of pLIV1 with lymph node involvement. European journal of cancer. 1994; 30A:675-678.

17. Taylor KM, Morgan HE, Johnson A, Hadley LJ, Nicholson RI. Structure-function analysis of LIV-1, the breast cancerassociated protein that belongs to a new subfamily of zinc transporters. The Biochemical journal. 2003; 375:51-59.

18. Helleman J, Jansen MP, Ruigrok-Ritstier K, van Staveren IL, Look MP, Meijer-van Gelder ME, Sieuwerts AM, Klijn JG, Sleijfer S, Foekens JA, Berns EM. Association of an extracellular matrix gene cluster with breast cancer prognosis and endocrine therapy response. Clinical cancer research. 2008; 14:5555-5564.

19. Wang K, Andresen Eguiluz RC, Wu F, Seo BR, Fischbach C, Gourdon D. Stiffening and unfolding of early depositedfibronectin increase proangiogenic factor secretion by breast cancer-associated stromal cells. Biomaterials. 2015; 54:63-71.

20. Oida K, Matsuda A, Jung K, Xia Y, Jang H, Amagai Y, Ahn G, Nishikawa S, Ishizaka S, Jensen-Jarolim E, Matsuda H, Tanaka A. Nuclear factor-kB plays a critical role in both intrinsic and acquired resistance against endocrine therapy in human breast cancer cells. Scientific reports. 2014; 4:4057.

21. McShane LM, Altman DG, Sauerbrei W, Taube SE, Gion M, Clark GM and Statistics Subcommittee of the NCIEWGoCD. REporting recommendations for tumour MARKer prognostic studies (REMARK). European journal of cancer. 2005; 41:1690-1696.

22. http://medgen.ugent.be/genorm

23. Mendiola M, Barriuso J, Marino-Enriquez A, Redondo A, Dominguez-Caceres A, Hernandez-Cortes G, PerezFernandez E, Sanchez-Navarro I, Vara JA, Suarez A, Espinosa E, Gonzalez-Baron M, Palacios J, Hardisson D. Aurora kinases as prognostic biomarkers in ovarian carcinoma. Human pathology. 2009; 40:631-638.

24. Garcia-Fernandez E, De Diego JI, Collantes-Bellido E, Mendiola M, Prim MP, Perez-Fernandez E, Miguel-Martin M, Nistal M, Hardisson D. Aurora B kinase expression in laryngeal squamous cell carcinoma and its prognostic implications. Histopathology. 2011; 58:368-376. 